mt. La FMH a élaboré en étroite collaboration avec l'Association suisse des médecins agréés travaillant en cliniques privées (ASMI/SVBP) un tarif de l'activité hospitalière dans l'assurance obligatoire des soins dédié aux médecins agréés. Le tarif, basé sur TARMED, intègre les résultats du recensement des frais de cabinet répertoriés pour les années 2000 et 2001 . Les principes de ce tarif sont présentés dans l'article suivant. Le Président de la FMH et l'ASMI ouvrirons sans délai des négociations avec les assureurs, afin de pouvoir introduire ce tarif dans les plus brefs délais aussi bien dans le domaine accident que maladie.

\title{
Le médecin agréé dans l'assurance sociale
}

\author{
B. Burri ${ }^{a}$, H. H. Brunner ${ }^{b}$
}

\section{Situation}

L'introduction imminente du TARMED dans le domaine ambulatoire des établissements hospitaliers qu'ils soient publics ou privés, plus précisément l'application des conventions cadre l'accompagnant, a fait apparaître au grand jour la situation difficile du médecin agréé, que d'aucuns ont stigmatisée depuis plusieurs années, sans que jusqu'ici, une solution raisonnable n'ait pu être trouvée. La difficulté rencontrée par les médecins agréés en milieu hospitalier est générée par une lacune dans la LAMal, qui, par ailleurs, n'est pas comblée dans la $2^{\mathrm{e}}$ révision en cours. Pour mémoire, on entend par médecin agréé un médecin ayant une activité d'une part en cabinet et d'autre part en établissement hospitalier privé ou public avec lequel il n'a pas de relation salariale, assurant ainsi lui seul son assurance vieillesse et survivants.

L'article 35 LAMal fait l'inventaire des fournisseurs de prestations admis à pratiquer à la charge de l'assurance obligatoire des soins. On y trouve séparément sous le point «a. les médecins» et sous le point «h. les hôpitaux». Les conventions tarifaires concernant la pratique médicale en cabinet, l'un des pans de l'activité du médecin agréé, sont conclues entre assureurs et associations de médecins selon l'art. 48 LAMal. C'est au travers de cet article que se trouve réglée sous l'égide de la société médicale cantonale la convention tarifaire de la pratique en cabinet en ce qui concerne la maladie alors que la FMH est responsable des conventions fédérales (AA, AM, $\mathrm{AI})$. La rémunération de l'autre pan, c'est-à-dire l'activité hospitalière, est définie à l'art. 49 LAMal qui stipule que «pour rémunérer le traitement hospitalier, y compris le séjour à l'hôpital, les parties à une convention conviennent de forfaits». C'est ici que se trouve le nœud car la lecture de cet article par les établissements hospitaliers et les assureurs est telle que les associations ou groupements de médecins sont écartés des négociations tarifaires. La Société vaudoise de médecine a été sensible à cette mise à l'écart du corps médical et demandé en conséquence en 2001 au Prof. J.-L. Duc un avis de droit sur la question «si une association de médecins peut faire partie à une convention tarifaire hospitalière». Le statut des médecins à l'hôpital est ici déterminant selon Duc: «L'article 49 LAMal permet aux médecins - et même elle leur impose d'être partie à une convention tarifaire par l'intermédiaire de leur organisation professionnelle lorsqu'ils ne sont pas salariés de l'hôpital et qu'il s'agit de fixer leur rétribution pour leur activité en division commune pour le compte de l'assurance obligatoire des soins; par contre la loi ne permet en revanche pas aux médecins de participer à une convention tarifaire lorsqu'ils ont le statut de salarié de l'hôpital.» En bref, l'établissement hospitalier convient seul ses forfaits avec les payeurs lorsque ses médecins sont ses salariés alors que cela ne lui est pas possible, qu'il soit un établissement public ou privé, lorsqu'il s'agit de l'activité hospitalière de ses médecins agréés. Le faire léserait de plus le droit suisse où un contrat à charge d'un tiers - car ce serait le cas - n'existe pas.

La décision du Conseil fédéral du 6 janvier 2002 (KV 245) à propos de la fixation du tarif pour une division commune d'une clinique privée confirme les éléments ci-dessus lorsqu'il dit: «L'art. 49 al 1 LAMal n'oblige pas à la fixation d'un forfait journalier complet incluant toutes les prestations: la fixation d'un forfait hospitalier journalier et d'un forfait par cas pour les honoraires des médecins est conforme à la loi.» Il appartient donc aux associations réunissant les médecins agréés de convenir de forfaits avec les assureurs pour leur activité hospitalière dans l'assurance obligatoire des soins. Le comité des Cliniques privées suisses (CPS) a admis ces éléments et recommandé à ses membres de laisser aux 
groupements des médecins agréés leur responsabilité lors des négociations tarifaires. Les hôpitaux publics doivent s'engager dans la même voie lorsqu'il s'agit de leurs médecins non salariés.

La perspective de l'introduction du TARMED dans le domaine hospitalier a cristallisé la lacune de la LAMal à l'égard des médecins agréés, d'autant plus que celui-ci ne peut y remédier, bien au contraire, car là aussi, le statut particulier du médecin agréé a été omis. Ainsi, la FMH, prenant conscience de la situation défavorable dans laquelle une partie non négligeable de ses membres allait se trouver et ayant reconnu leur importance et leur qualité dans l'organisation sanitaire suisse, a décidé en étroite collaboration avec l'Association suisse des médecins agréés travaillant en cliniques privées (ASMI/SVBP) d'élaborer un tarif de l'activité hospitalière dans l'assurance obligatoire des soins dédié aux médecins agréés.

Ce tarif, exprimé sous forme de forfaits et en francs, est une émanation de la structure tarifaire TARMED. Pendant son activité hospitalière, le cabinet médical doit être disponible même en l'absence du médecin agréé. Le coût de cette situation doit être pris en compte par ce tarif. C'est pourquoi l'ASMI a mis sur pied un recensement des frais de cabinet des médecins agréés des années 2000 et 2001, qui a été validé par une autorité indépendante en la personne du Prof. G. Neubauer de l'Institut de l'économie de la santé à Munich.

\section{Recensement et évaluation des frais de cabinet des médecins agréés en Suisse}

Au cours de l'été 2003, l'Association suisse des médecins indépendants travaillant en cliniques privées (ASMI), en collaboration avec la FMH, a organisé un vaste recensement des frais de cabinet des médecins agréés pratiquant en Suisse dans le but de recueillir les critères économiques de l'activité particulière du médecin agréé.

Plus de 1100 données obtenues sur les années 2000 et 2001 ont pu ainsi être évaluées. Elles correspondent aux rubriques composant un plan comptable usuel et sont: les frais de personnel, de locaux, d'installation et d'entretien du cabinet, de matériel, d'assurances (y compris AVS et $3^{\text {e }}$ pilier), de comptabilité, de véhicule, de formation postgraduée, d'investissement, de frais divers, de dépenses en capital, d'autres frais et de redevances fixes versées à l'hôpital indépendamment des prestations fournies.
Seuls les questionnaires complètement remplis ont été retenus parmi un nombre élevé de réponses - ce qui a été réjouissant - et validés, mettant à jour des variations significatives entre les différentes disciplines aussi bien vers le haut que vers le bas. Le calcul de la médiane a permis d'écarter les valeurs extrêmes. La médiane des frais globaux d'un cabinet de médecin agréé en Suisse s'élève à:

Fr. 358204.-- pour l'année 2000 et à

Fr. 376325.- pour l'année 2001.

Le résultat global mais également les données par discipline et par rubriques comptables ont été soumis à un expert en économie de la santé en la personne du Prof. G. Neubauer de l'Université de Munich, qui les a vérifiés puis validés. L'étude des valeurs obtenues a révélé une grande similitude avec celles émanant des cabinets des médecins agréés de la partie ouest de l'ancienne Allemagne.

Les résultats de ce recensement ont été utilisés pour construire, selon des critères d'économie d'entreprise, un tarif dédié aux médecins agréés qui ont à la fois une activité médicale indépendante en cabinet et en établissement hospitalier.

\section{Tarif de I'activité hospitalière du médecin agréé dans l'assurance de base}

1. Il s'agit de forfaits par intervention pratiquée en milieu hospitalier et par un médecin agréé (opérateur, anesthésiste, assistant et médecin consultant selon nécessité) calculés par cas, comprenant le rapport opératoire. Les visites font l'objet également de forfaits. Les interventions pour complications, à l'exception des hémorragies dans les 24 heures, sont rémunérées selon des forfaits distincts. Le cumul d'interventions est possible pour autant qu'il s'agisse de voies d'abord ou de compartiments anatomiques différents.

2. Les interventions fréquemment pratiquées se trouvent dans ce tarif qui ne peut cependant faire état de tous les cas particuliers. Ces derniers sont soumis à une réglementation particulière ou le cas échéant à une commission dite de clearing pour la détermination des honoraires. Les décisions ou interprétations de cette commission, rendues anonymes, pourront être consultées.

3. Le tarif, basé sur la nomenclature et le minutage du TARMED, intègre les résultats du recensement des frais de cabinet répertoriés pour les années 2000 et 2001. 
4. Les forfaits, exprimés en francs, servent à rémunérer les prestations médicales des médecins agréés lors de leur activité ambulatoire et stationnaire en établissement hospitalier à charge de l'assurance sociale dans toute la Suisse.

5. Les interventions de chaque discipline sont réparties actuellement dans 21 forfaits dont le plus élevé est au maximum de Fr. 4000.pour l'opérateur et de Fr. 2500.- pour l'anesthésiste. L'assistant, lorsqu'il s'agit d'un médecin agréé (donc avec un cabinet à charge) perçoit le $70 \%$ alors que, lorsqu'il ne l'est pas agréé, le $30 \%$ du forfait considéré.

Tableau 1

Quelques exemples.

\begin{tabular}{lll}
\hline Forfait & Montant (Fr.) & Intervention \\
\hline 2 & 300 & Correction orteil en marteau; Cytoscopie \\
\hline 3 & 400 & Curetage \\
\hline 4 & 500 & Cure tunnel carpien; Coloscopie \\
\hline 5 & 600 & Thoracoscopie \\
\hline 6 & 700 & Appendicectomie \\
\hline 7 & 800 & $\begin{array}{l}\text { Amygdalectomie et adénoïdectomie; Op. cataracte unilatérale; } \\
\text { Tonsillectomie }\end{array}$ \\
\hline 8 & 900 & $\begin{array}{l}\text { Césarienne } \\
\text { Cholécystectomie; Crossectomie et stripping unilatéral et varices; } \\
\text { TURP }\end{array}$ \\
\hline 10 & 1000 & Prothèse de hanche \\
\hline 16 & 1250 & Pontage aorto-bi-fémoral \\
\hline 21 & 4000 & Triple pontage coronarien \\
\hline & \\
La construction de ce tarif repose sur une mécanique extrêmement complexe qu'il n'est pas \\
judicieux de détailler ici.
\end{tabular}

\section{Conclusions}

En un laps de temps extrêmement court, les délégués des différentes sociétés ont transmis les modifications concernant leur discipline et donné sans réticence leur aval à ce tarif. Le Comité central de la FMH à qui ce projet a été présenté, reconnaissant le bien-fondé de celui-ci, fort du soutien sans réticence des médecins agréés des différentes disciplines, a chargé unanimement son Président et l'ASMI d'ouvrir sans délai des négociations avec les assureurs accident et maladie, ce qui devrait se faire encore cette année.

Le but principal d'un tarif de l'activité hospitalière en établissement hospitalier privé ou public dédié aux médecins agréés dans le domaine de l'assurance obligatoire des soins est de combler la lacune de la LAMal et de rendre aux médecins la responsabilité de la négociation de leurs honoraires avec les assureurs maladie mais également dans le domaine de l'accident.

Pour les assureurs, la création de forfaits en utilisant la structure tarifaire TARMED, réalisant un tarif uniforme de l'activité médicale hospitalière pour toute la Suisse et basé sur des critères d'économie d'entreprise, est à l'évidence un élément de simplicité et de transparence, qui a déjà trouvé par ailleurs un bon accueil parmi les médecins agréés des différentes disciplines médicales, bien que l'acceptation définitive soit entre les mains de l'assemblée générale de l'ASMI à laquelle le tarif définitif, accepté par les partenaires contractuels, sera soumis. Lors des négociations que vont engager la FMH et l'ASMI, il est à souhaiter que les partenaires sachent percevoir rapidement le bien-fondé et l'utilité de ce projet pour le service sanitaire suisse afin de pouvoir l'introduire dans les plus brefs délais aussi bien dans le domaine accident que maladie. 